\title{
Microstructure Analysis of Nanocrystalline Powders by X-ray Diffraction
}

\author{
D. LOUËr AND N. AUdEBRAND \\ Laboratoire de Chimie du Solide et Inorganique Moléculaire (UMR CNRS 6511) \\ Institut de Chimie, Université de Rennes \\ Av. du Général Leclerc, 35042 Rennes cedex, France
}

\begin{abstract}
The use of X-ray diffraction line profile analysis for the study of nanocrystalline powders is described. The fundamentals of the theory are presented in terms of crystallite/domain size, size distribution, lattice distortion, dislocation density and stacking faults. Line profile parameters and the methods of pattern fitting introduced to overcome the diffraction-line overlap problem are described. The approaches based of the integral breadth of the measured line profiles and the Fourier method are discussed. In addition, simplified approaches are also commented. Representative examples are selected to illustrate various cases of microstructure, such as nanomaterials with strain-free spherical nanocrystallites, strain-free crystallites with anisotropic crystallite shape, anisotropic crystallites with microstrains and spherical crystallites with dislocation densities and crystallite size distributions.
\end{abstract}

PACS numbers: 61.10.Dp, 61.10.Nz, 61.46.+w, 61.72.Dd, 61.72.Lk, 61.72.Mm

\section{Introduction}

The investigation of size dependent properties of nanoscale materials has received much interest in the recent years (see, for instance, Refs. [1-3]). Among the techniques used to characterize nanomaterials the powder diffraction method provides information on the size of crystallites (domains over which diffraction is coherent) and structure mistakes. The method, introduced long ago with the Scherrer equation, has progressed considerably with pattern fitting approaches (see, for instance, Refs. [4, 5]). In particular, the interpretation of anisotropic line broadening in terms of crystallite shape [6] or dislocation density [7] has been described. Recent trends deal with pattern modelling using physical-parameters 
based models [8]. The traditional approaches of line broadening analysis can usually be applied for crystallite sizes in the approximate range $3-100 \mathrm{~nm}$, though the higher limit can be extended by increasing the resolution of the instruments, e.g. with ultrahigh resolution diffractometers available with synchrotron X-rays. Below $\approx 3 \mathrm{~nm}$ the diffraction lines broaden considerably and, then, pattern modelling approaches are useful. In this last range of sizes the study of supported catalysts by diffraction is also of particular interest [9]. Most of modern advances have been considered at length in the recent book Defect and Microstructure Analysis by Diffraction [10].

Microstructural effects contributing to diffraction line broadening include the small size of crystallites, size distributions, stacking faults, and lattice distortions. The size effect is related to an apparent size of the crystallites, which includes their true size, and a possible fictitious size on certain $h k l$ planes if stacking faults are present. Lattice distortion corresponds to small changes in $d_{h k l}$ spacings. These changes, generally expressed by the relative deformation $\Delta d / d$, can be due to dislocations, punctual defects in nonstoichiometric materials, $d$-spacing fluctuations in layered materials, or chemical composition gradients. Line broadening due to lattice distortion is generally known as the strain effect. The paper is organised as follows. Section 2 deals with the characteristics of diffraction lines and modern fitting techniques. In the subsequent sections the basic theory of diffraction by nanocrystallites (Sec. 3) and simplified analyses (Sec. 4) are considered. Finally, representative applications to powder nanomaterials are reported in Sec. 5.

\section{Diffraction line profiles and pattern fitting}

Observed diffraction line profiles $h(x)$ are the convolution of physical profiles $f(x)$, due to the sample microstructure, and instrumental profiles $g(x)$, including wavelength dispersion, i.e. $h(x)=f(x) * g(x)$. Deconvolution methods (see Ref. [10]) must be applied to correct observed profiles $h(x)$ from the instrumental contribution. Among them, the method based on the multiplicative property of the related Fourier transforms, $H(t)=F(t) G(t)$, is often used to obtain $f(x)$ and its Fourier coefficients. The $f(x)$ profiles result themselves from the convolution of components arising from the microstructure contributions, e.g. $f(x)=f^{\mathrm{S}}(x) * f^{\mathrm{D}}(x) * f^{\mathrm{F}}(x)$, where $f^{\mathrm{S}}(x), f^{\mathrm{D}}(x), f^{\mathrm{F}}(x)$ stand for size, distortion, and stacking faults effects, respectively.

Line profiles are characterized by a position parameter, e.g. the peak position defined by the angle $2 \theta_{0}$ where the profile intensity is maximum $\left(I_{0}\right)$ and a measure of intensity dispersion or line broadening, such as the full width at half maximum $F W H M$ and the integral breadth $\beta\left[=\int I(2 \theta) \mathrm{d}(2 \theta) / I_{0}\right]$ which has considerable theoretical advantages. A useful parameter is the line shape expressed as $\phi=$ $F W H M / \beta$. The breadths are measured experimentally in degrees $2 \theta$, but for a physical interpretation it is preferable to use reciprocal units $\left(\mathrm{nm}^{-1}\right)$, i.e. $\beta^{*}=$ 
$\left(\beta_{2 \theta} \cos \theta\right) / \lambda$. The elimination of the instrumental contribution from $\beta_{h}$ requires assumptions about line profile shapes, e.g. for Cauchy shapes $\beta_{h}=\beta_{f}+\beta_{g}$ and for Gauss shapes $\beta_{h}^{2}=\beta_{f}^{2}+\beta_{g}^{2}$. If the profiles are adequately described by Voigt functions (see below) a precise evaluation of $\beta_{f}$ can be carried out. Within the context of the Fourier analysis, the $f(x)$ is given by a Fourier series

$$
f(x)=\sum_{-\infty}^{+\infty}\left[A_{n} \cos (2 \pi n x)+B_{n} \sin (2 \pi n x)\right],
$$

where $x$ is the reciprocal space variable and $n$ - the harmonic number. In practice, $x$ takes the form $\left(s-s_{0}\right) / \Delta s$, where $s=2 \sin \theta / \lambda, s_{0}=2 \sin \theta_{0} / \lambda=1 / d_{h k l}$, $\Delta s=\left(2 \sin \theta_{2}-2 \sin \theta_{1}\right) / \lambda,\left(\theta_{1}, \theta_{2}\right)$ is the angular range over which the profile is defined, $\theta_{0}$ and $d$ are the angle and spacing of the Bragg reflection $h k l$.

Pattern modelling techniques have been introduced to overcome the problem of line overlap which results from the rotational projection of the nodes of the reciprocal lattice onto one-dimensional pattern. Two different approaches are currently applied, the pattern decomposition method, for which no structural information is required, and the Rietveld method which includes the refinement of atomic coordinates. The procedure consists of fitting, usually with a least squares refinement, a calculated model to the whole observed diffraction pattern. These techniques can be used to restore the individual line profiles, provided that the degree of line overlap is not too severe. The calculated intensity $y\left(x_{i}\right)$ at point $x_{i}$ is expressed as a function of the integrated intensity $I_{k}$ of reflection $k$ and a normalised analytical function $\Phi$ is used to model individual line profiles

$$
y_{\text {cal }}\left(x_{i}\right)=\sum_{k} I_{k} \Phi\left(x_{i}-x_{k}\right)+b\left(x_{i}\right)
$$

where $b\left(x_{i}\right)$ is the intensity of the background and the sum is over all reflections contributing to the intensity at $x_{i}$. The most commonly used line shape functions $\Phi$ are derived from the Gauss $(G)$ and the Cauchy $(C)$ functions (for a detailed description, see Appendix 1 in Ref. [4]). The pseudo-Voigt $[\eta C+(1-\eta) G]$ is the sum of $G$ and $C$ components, in a proportion defined by $\eta(\eta=1$ for $C$ and 0 for $G$ ). The Pearson VII function is $(C)^{m}$, where the exponent $m$ is the line shape parameter ( $m=1$ for $C$ and $m=\infty$ for $G$ ). The Voigt is a convolution of $C$ and $G$ components whose limits of application are defined by the shape parameter $\phi$, i.e. 0.6366 for $C$ and 0.9394 for $G$. Fitting techniques and line broadening analysis (Fourier and integral breadth methods) are often combined to overcome line overlap problems $[11,12]$.

In the Rietveld method $I_{k}$ is expressed as a function of the square of the structure-factor modulus $\left|F_{k}\right|^{2}$ and the atomic coordinates are adjusted to get the best fit to observed data. The angular dependence of line widths is described by a quadratic form, i.e. $F W H M^{2}=U \tan ^{2} \theta+V \tan \theta+W$, where $U, V$ and $W$ are refined parameters. If the diffraction lines are physically broadened, such angular dependence is valid for isotropic microstructual properties. If a pseudo-Voigt func- 
tion is used, the Gauss components $F W H M_{G}$ follow the above equation, while the Cauchy components can be written as $F W H M_{C}=X \tan \theta+Y / \cos \theta$ [13]. It contains explicitly both a term with $\cos ^{-1} \theta$ and a one with a $\tan \theta$ dependence, which are related to the size and distortion effects, respectively. More sophisticated phenomenological models have been reported for anisotropic cases [14] and the Rietveld method has also been discussed in microstructural terms using integral breadth [15] and Fourier approaches [16].

\section{Diffraction by nanoscale and imperfect crystallites}

The basic expression for a diffraction line profile resulting from small and distorted crystallites is given by $[17,18]$

$$
f(s)=\int V(t) y(t) \exp (-2 \pi \mathrm{i} s t) \mathrm{d} t
$$

where $V(t)$ is conveniently represented as the volume common to the crystallite and its double, displaced a distance $t$ in the direction of the diffraction vector, and $y(t)$ is the average value of the product $F F^{*}$ of complex structure-factors of pairs of cells separated by a distance $t$ in the diffraction vector direction. $V(t) y(t)$ is then the Fourier transform of $f(s)$.

\subsection{Size effect}

For negligible distortion, $y(t)$ is a constant, the reciprocal of the initial slope of the volume function $V(t)$ is the Fourier apparent size $\varepsilon_{\mathbf{F}}\left[=-V(0) / V^{\prime}(t)_{t=0}\right]$ of the crystallite in the direction of the diffraction vector [19]. This is an area-weighted mean apparent size. The second derivative $V^{\prime \prime}(t)$ is proportional to the crystallite thickness distribution measured in the direction of the diffraction vector. There is a relation between $V(t)$ and the integral breadth $\beta^{\mathrm{S} *}$, i.e. $\beta^{\mathrm{S} *}=V(0) / \int V(t) \mathrm{d} t$, from which the integral-breadth apparent size $\varepsilon_{\beta}\left(=1 / \beta^{S *}\right)$ is derived. This is a volume-weighted average size. In order to make allowance for the variation of thickness within a crystallite in the direction of the diffraction vector some regular morphology must be assumed [20]. The simple case is the sphere for which the equivalent area-weighted and volume-weighted mean diameters are $\left\langle D_{\mathrm{A}}\right\rangle=3 \varepsilon_{\mathrm{F}} / 2$ and $\left\langle D_{\mathrm{V}}\right\rangle=4 \varepsilon_{\beta} / 3$, respectively. A useful model for anisotropic crystallite shapes, used in the examples discussed in the present overview, is the cylinder which can be conveniently applied from acicular to disk crystallite shapes [21].

\subsection{Distortion effect}

The interpretation of $y(t)$ is not so straightforward. In the Fourier approach the mean strain $e_{n}$ is defined as $\Delta L / L$, where $L\left(=n d_{h k l}\right.$ or, in practice, $n^{\prime} / \Delta s$ according to the period $d^{*}$ or $\Delta s$ over which the Fourier coefficients are calculated [22]) is the length between pairs of undistorted cells separated by $n$ cells and $\Delta L$ 
is the distance change due to distortion. In the Warren-Averbach-Bertaut [22], the complex coefficients $C_{n}(l)\left[=A_{n}(l)+\mathrm{i} B_{n}(l)\right]$ for a reflection $l$ are expressed by the product of a size real coefficient $A_{n}^{\mathrm{S}}$, independent of the order $l$, and a distortion order-dependent complex coefficient $C_{n}^{\mathrm{D}}(l)\left[=A_{n}^{\mathrm{D}}(l)+\mathrm{i} B_{n}^{\mathrm{D}}(l)\right]$. The two coefficients can be separated if, at least, two orders are available. This can be carried out from the plots $\ln A_{n}(l)$ versus $l^{2}$, which result from series expansions of cosine and logarithm terms

$$
\ln A_{n}(l)=\ln A_{n}^{\mathrm{S}}-2 \pi^{2} l^{2} n^{2}\left\langle e_{n}^{2}\right\rangle .
$$

The procedure is valid for small values of $l$ and $n$ (or $L$ ). It is exact if Gaussian distributions of $e_{n}$ occur. In Eq. (4), $e_{n}$ is the root-mean-square (r.m.s.) strain. After separation, the size coefficients $A_{n}^{\mathrm{S}}$ can be interpreted as explained in Sec. 3.1, though for numerical calculations Fourier transforms are substituted by the Fourier series.

The integral breadth of $f(x)$ provides another estimation of microstrain. An apparent strain parameter has been introduced, i.e. $\eta_{\beta}=2 \beta^{\mathrm{D} *} / d^{*}=\beta_{2 \theta}^{\mathrm{D}} \operatorname{cotan} \theta$, where $\beta^{\mathrm{D} *}$ is the distortion integral breadth $[23,24]$. Two simple strain models for which the apparent strain is related to the true distortion have been discussed. In the first model, the true strain $\hat{e}\left(=\Delta d / d=\eta_{\beta} / 4\right)$ is based on the assumption that the Bragg equation is valid over the angular range corresponding to $d \pm \Delta d$. In the second model, the strain is defined by the r.m.s. strain $\tilde{e}=\left\langle e^{2}\right\rangle^{1 / 2}=$ $\eta_{\beta} /\left[2(2 \pi)^{1 / 2}\right] \cong \eta_{\beta} / 5$.

\subsection{Anisotropic microdistortion and dislocations}

Anisotropic microdistortions have been interpreted with the dislocation model based on the r.m.s. strain of dislocated crystals [7]. In this model of $\left\langle e_{n}^{2}\right\rangle$, the contribution to strain-induced line broadening depends on the relative orientations of the line and Burgers vectors of the dislocations and the diffraction vector, similar to the contrast effect of dislocations in transmission electron microscopy. This anisotropic effect is taken into account with contrast factors $C$, which have been calculated on the basis of the crystallography of dislocations and the elastic constants for cubic [25] and hexagonal [26] materials. According to Krivoglaz [27] and Wilkens [28], for small values of $L$, the microdistortion term $\left\langle e_{n}^{2}\right\rangle$ in the Warren-Averbach approach (Eq. (4)) is expressed as a function of dislocation parameters, namely the dislocation density $(\rho)$, the modulus of the Burgers vector $(b)$ and the effective outer cut-off radius of dislocations $\left(R_{e}\right)$, i.e. $\left\langle e_{n}^{2}\right\rangle=\left(\rho \bar{C} b^{2} / 4 \pi\right) \ln \left(R_{e} / L\right)$. For cubic materials, the average dislocation factors can be written as $\bar{C}=\bar{C}_{h 00}\left(1-q H^{2}\right)$ [25], where $\bar{C}_{h 00}$ is the average dislocation contrast factor for the $h 00$ reflections, $q$ is a parameter depending on the elastic constants and the nature of the dislocations and $H^{2}=\left(h^{2} k^{2}+h^{2} l^{2}+k^{2} l^{2}\right) /\left(h^{2}+k^{2}+l^{2}\right)^{2}$. The values of $C_{h 00}$ can be obtained from tables [29]. In particular, $\bar{C}_{h 00}$ and $q$ have been estimated for dislocations 
with the Burgers vectors $a / 2\langle 110\rangle$ and $a / 2\langle 111\rangle$. The density of dislocations is then calculated from the modified Warren-Averbach method, after insertion in Eq. (4) of the contrast factor

$$
\ln A(L)=\ln A^{\mathrm{T}}(L)-\left(\pi b^{2} / 2\right) \rho L^{2} \ln \left(R_{e} / L\right)\left(d^{* 2} \bar{C}\right)+\mathrm{O}\left(d^{* 4} \bar{C}^{2}\right),
$$

where $O$ stands for higher order terms in $d^{* 2} \bar{C}$. Analyses carried out over all reflections can be carried out with the program MWP-fit [30]. The Fourier transforms of each profile are fitted simultaneously using a linear least-squares algorithm. The interpretation is extended to the crystallite size distributions assuming lognormal functions, which have been proved to occur in a loose powder of $\mathrm{CeO}_{2}$ [31].

\subsection{Stacking faults}

The effect of stacking faults on line broadening has been discussed by Guinier [17], Wilson [18] and Warren [22]. The diffracting domains are limited by the faults. The integral breadth is inversely proportional to the mean distance between $h k l$ planes concerned by the mistakes. This effect is similar to size broadening, though line broadening is selective, i.e. it depends on $h k l$. Various types of faults can be considered, e.g. deformation faults, growth faults, and twin faults. Stacking faults have three main effects on line profile: the apparent size is modified, the deformation faults introduce a shift of certain reflection in cubic symmetry and asymmetry is observed in case of twinning. The case of hexagonal symmetry is simpler for faults in planes perpendicular to the $c$ axis. The hexagonal close packed sequence $\mathrm{ABA} \ldots$ is replaced by the plane series $\mathrm{ABABAB} \perp \mathrm{CBCBCB}$... where a fault is intercalated. Some reflections are symmetrically broadened without shift of their position. As a consequence, the integral breadths, expressed as a function of the stacking fault probability $\alpha$ and the angle $\phi_{z}$ between the diffraction vector and $c$ axis, follow particular conditions on $h k l$ forming three groups of reflections, i.e. $h-k=3 n$ and $h k 0, \beta^{\mathrm{F} *}=0 ; h-k=3 n \pm 1, \beta^{\mathrm{F} *}=\alpha \cos \phi_{z} / 2 c$ for $l$ even and $\beta^{\mathrm{F} *}=3 \alpha \cos \phi_{z} / 2 c$ for $l$ odd $[18,32]$.

\section{Simplified analysis of the microstructure}

A simplified analysis of diffraction line broadening was introduced through the so-called Williamson-Hall plots [33] in which $\beta_{f}^{*}$ (or $F W H M_{f}^{*}$ ) is plotted for all $h k l$ reflections as a function of $d^{*}(=2 \sin \theta / \lambda)$. This simple representation assumed that all profiles have a Cauchy shape. The method should not be used quantitatively, but it gives a useful overview of the microstructural effects at the origin of line broadening and, generally, it suggests the appropriate subsequent analysis to carry out. In practice, several models are available for simplified analysis, depending on the profile shape assumptions for the size and strain line profiles, namely Cauchy-Cauchy $\beta_{f}^{*}=\varepsilon_{\beta}^{-1}+2 e d^{*}$, Gauss-Gauss $\beta_{f}^{* 2}=\left(\varepsilon_{\beta}^{-1}\right)^{2}+4 e^{2} d^{* 2}$ and Cauchy-Gauss $\beta_{f}^{* 2}=\left(\varepsilon_{\beta}^{-1}\right) \beta_{f}^{*}+4 e^{2} d^{* 2}$, where $e$ is the real component of strain. 
The interpretation of $e$ depends on the strain model selected, as discussed in Sec. 3.2. From the Cauchy-Gauss model, which is an approximation of the Voigt function, an "average size-strain" plot $\left(\beta_{f}^{*} / d^{*}\right)^{2}$ versus $\beta_{f}^{*} /\left(d^{*}\right)^{2}$ can be used for the evaluation of strain (intercept) and size (slope) for isotropic cases [20]. In this interpretation the Cauchy component $\beta_{f C}^{*}$ is attributed to size and the Gauss component $\beta_{f \mathrm{G}}^{*}$ to strain.

For anisotropic microdistortion a modified Williamson-Hall plot, based on the breadths $B_{f}^{*}\left(\beta_{f}^{*}\right.$ or $\left.F W H M^{*}\right)$, taking into account the contrast factors due to dislocations has been introduced $[7,34]$ :

$$
B_{f}^{*}=(1 / \varepsilon)+\left(\pi M^{2} b^{2} / 2\right) \rho^{1 / 2}\left(d^{*} \bar{C}^{1 / 2}\right)^{2}+\mathrm{O}\left(d^{*} \bar{C}^{1 / 2}\right)
$$

where $\varepsilon\left(\varepsilon_{\beta}\right.$ or $\left.\varepsilon_{F W H M} / 0.9\right)$ is the mean apparent size and $M$ - a constant dependent on the effective outer cut-off radius of dislocations (see Sec. 3.3).

\section{Applications and discussion}

\subsection{Isotropic nanocrystallites: $\mathrm{CeO}_{2}$}

Several examples of $\mathrm{CeO}_{2}$ powders with isotropic diffraction line broadening have been reported. They were obtained from soft thermal decomposition of inorganic precursors $[35,36]$ or from gas condensation [37]. In the case of $\mathrm{CeO}_{2}$ obtained thermally in the range $600-850^{\circ} \mathrm{C}$ from hydrated ceria precipitated from a sulfate solution [36], the Williamson-Hall plots have a zero slope, whatever the sample preparation temperature, which is an indication of strain-free spherical crystallites on average with volume-weighted diameters $\left\langle D_{\mathrm{V}}\right\rangle$ in the range $26.3(8)-116.7(8.7) \mathrm{nm}$. Fourier transforms are then superposed, within the experimental error, for $15 \mathrm{hkl}$ reflections, with area-weighted diameters $\left\langle D_{\mathrm{A}}\right\rangle$ in the range $19.5(1.2)-92.7(13.1) \mathrm{nm}$. The difference in the two diameter values is due to a crystallite-size distribution, as shown by the ratio of apparent sizes $\left\langle\varepsilon_{\beta}\right\rangle /\left\langle\varepsilon_{\mathrm{F}}\right\rangle$ in the range $1.52-1.42$, while for homodispersed nanocrystallites the ideal ratio is 1.125 .

\subsection{Anisotropic strain-free crystallites: ex-hydroxynitrate $Z n O$}

The Willliamson-Hall plot (Fig. 1a) of a sample obtained at $210^{\circ} \mathrm{C}$ from the decomposition of the hydroxynitrate [6], shows that $\beta_{f}^{*}$ is the same, within the experimental error, for different orders of $h 00, h 0 h$ and $00 l$. This is an indication about strain-free crystallites. The intercept for $00 l$ reflections is smaller than that observed for $h k 0$ reflections, indicating a prismatic crystallite shape with a diameter smaller than the height. An average circular cross-section is deduced from the similar $\beta_{f}^{*}$ values obtained from $h 00$ and $h k 0$ lines. These features are supported by the behaviour of $\beta_{f}^{*}$ for $10 l$ lines, for which the angle $\phi_{z}$ between the diffraction vector and base plane increases from 0 to $\pi / 2$. From a least squares analysis the crystallite shape model shown in Fig. 1b has been obtained. It is characterized by 
a height of $27(6) \mathrm{nm}$ and a diameter of 18(1) $\mathrm{nm}$. The observed apparent sizes $\varepsilon_{\beta}$ are indicated by arrows for various crystallographic directions. This is an example of a three-dimensional representation of crystallite shape.
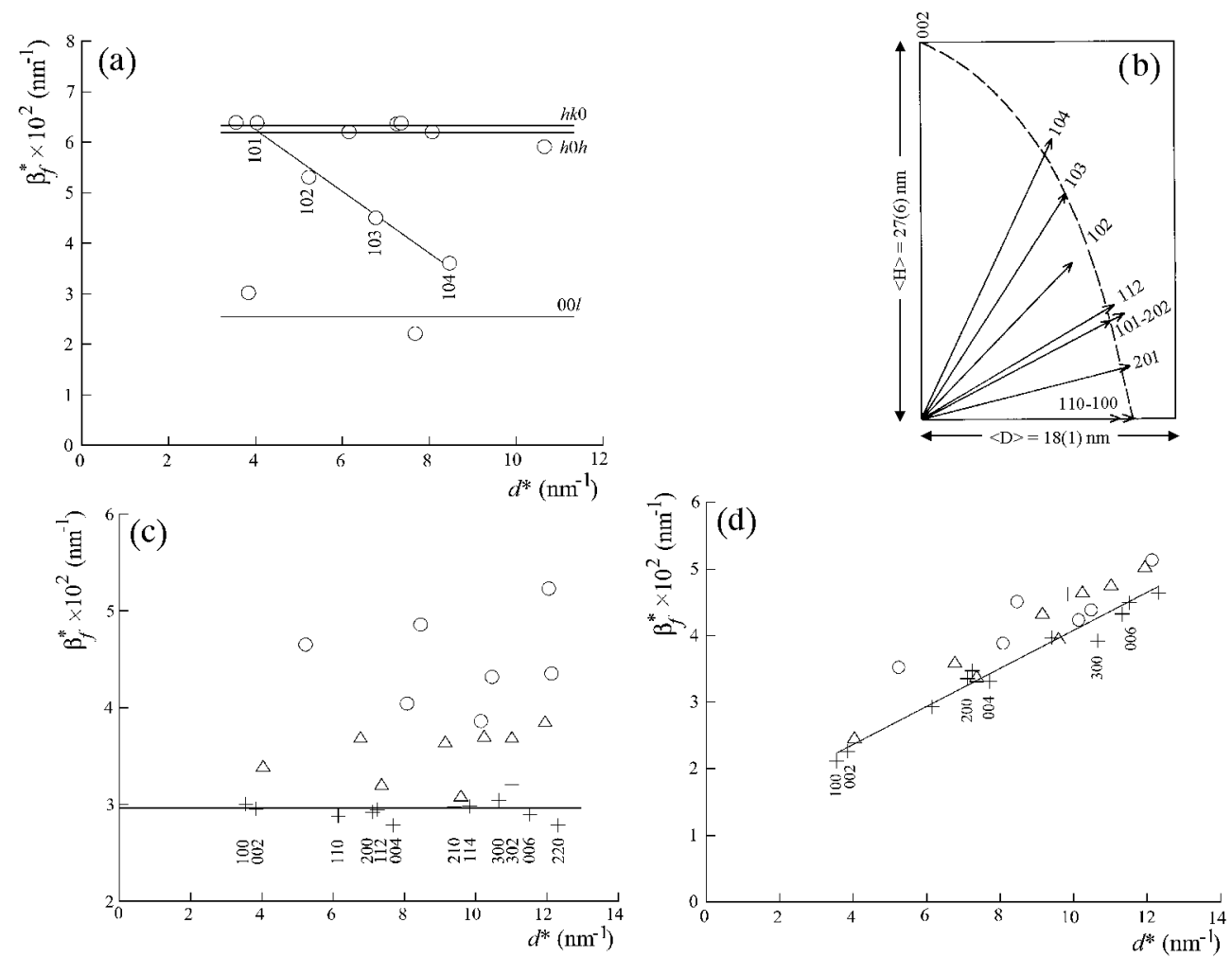

Fig. 1. Microstructure analysis of nanocrystalline ZnO. (a) Williamson-Hall plot for strain-free ex-hydroxynitrate $\mathrm{ZnO}$; (b) section of the cylindrical model for ex-hydroxynitrate $\mathrm{ZnO}$, obtained from least squares fit, showing the observed apparent sizes $\varepsilon_{\beta}$ shown by the length of the arrows in various $h k l$ directions, the broken line is the loci of the calculated apparent sizes (from Ref. [6]); (c) Williamson-Hall plot for ex-oxalate strain-free $\mathrm{ZnO}$ displaying stacking faults effects visible for two reflection groups $(\Delta, 0)$, according to the $h k l$ conditions listed in Sec. 3.4 (from Ref. [32]); (d) Williamson-Hall plot for ex-acetate $\mathrm{ZnO}$, showing the presence of size, strain and stacking faults (from Ref. [40]).

\subsection{Anisotropic crystallites with stacking faults: ex-oxalate ZnO}

The Williamson-Hall of $\mathrm{ZnO}$ obtained from an oxalate at $400^{\circ} \mathrm{C}$ is shown in Fig. 1c [38]. The scatter of integral breadths is interpreted by three groups of reflections according to $h k l$ as reported in Sec. 3.4. This is a typical example of 
stacking faults in a nanocrystalline hexagonal material. For the group of reflections ( $h k 0, h-k=3 n$ ), unaffected by the faults, the scatter of the breadths is almost negligible and the corresponding intercept suggests close sizes in the directions perpendicular to $(h 00)$ and $(00 l)$. The least squares analysis, based on the cylindrical model, gave a diameter of $40(1) \mathrm{nm}$ and a height of 33.6(1.2) nm. The calculated mean fault probability $\alpha=0.008$ was derived from the analysis of the two other reflection groups, i.e. the mean distance between faults is $31.6 \mathrm{~nm}$ or there is, on a verage, one fault per crystallite. The microstructure changes during initial crystallite growth of four nanocrystalline $\mathrm{ZnO}$ samples obtained from the hydroxynitrate-, oxalate-, acetate- and hydroxycarbonate-precursors have been thoroughly studied from line broadening analysis [38-40]. An example of Williamson-Hall plot for ex-acetate $\mathrm{ZnO}$ is shown in Fig. 1d. It is representative of combined size, strain, and stacking fault effects. From this study concepts on the microstructure dependence with the chemical nature of the precursors were derived.

\subsection{Anisotropic crystallites with distortion: $\mathrm{Ni}(\mathrm{OH})_{2}$}

The Williamson-Hall plot of a sample of hexagonal $\mathrm{Ni}(\mathrm{OH})_{2}[41]$, based on 24 reflections, is shown in Fig. 2a. For $00 l, h 0 h$, and $h 00$ reflections positive slopes are observed, indicating the presence of microdistortions. The non-zero intercept
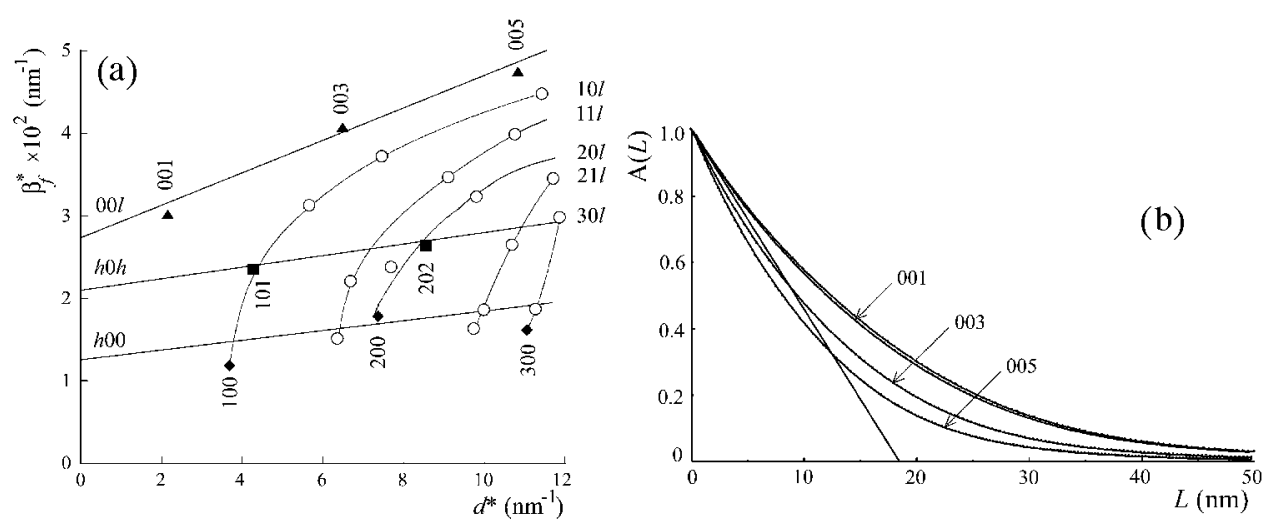

Fig. 2. Microstructure analysis of nanocrystalline $\mathrm{Ni}(\mathrm{OH})_{2}$ (from Ref. [41]). (a) Williamson-Hall plot displaying the anisotropy in crystallite shape and the presence of lattice distortion; (b) cosine Fourier coefficients for $00 l$ diffraction lines, demonstrating the order dependence of line broadening.

for the three families indicates the presence of a size effect. Moreover, the sequence $\beta^{*}(h 00, h k 0)<\beta^{*}(h 0 h)<\beta^{*}(00 l)$ can be interpreted from disk-shaped crystallites with the $c$ axis collinear to that of the disk. The integral breadths of groups of reflections $(10 l, 11 l),(20 l, 21 l)$ and $30 l$ follow a behaviour in accordance with the model. However, the presence of strains makes a bit more complicated the 
quantitative analysis from the integral breadth approach. The Fourier transforms were calculated for different orders $(001 / 003 / 005,100 / 200$, and 101/202) in order to separate size and strain effects. They are shown in Fig. $2 b$ for the reflections 001/003/005. From the initial slopes, the apparent sizes $18.4 \mathrm{~nm}, 54.5 \mathrm{~nm}$, and $26.2 \mathrm{~nm}$ were calculated in the directions perpendicular to (001), (100), and (101), respectively. The average parameters $\langle D\rangle=69.4 \mathrm{~nm}$ and $\langle H\rangle=18.4 \mathrm{~nm}$ were found. The apparent size $(23.8 \mathrm{~nm})$ calculated from these values in the direction perpendicular to (101) was in good accordance with the observed apparent size.

\subsection{Isotropic crystallites with dislocations: ex-ammonium-nitrate $\mathrm{Ce}_{2}$}

Williamson-Hall plots of samples of $\mathrm{CeO}_{2}$ obtained thermally in the range $600-850^{\circ} \mathrm{C}$, from hydrated ceria precipitated from an ammonia solution [36], revealed a significant anisotropic line broadening with an a verage positive slope. This is an interesting case of anisotropic distortion. This anisotropy can be seen, for example, on the breadth of line 111 greater than that of 200 , which corresponds to an elastic anisotropy, $A_{z}=2 c_{44} /\left(c_{11}-c_{12}\right)$, smaller than one. It has been treated and rationalized in terms of the dislocation model described in Sec. 3.3 [34]. The final results are shown in Fig. 3, i.e. the changes with temperature in dislocation densities and mean values of the size distribution functions (Fig. 3a) and the modifications of the size distribution functions with temperature (Fig. 3b). It is seen that the size distributions shift to larger values with decreasing densities as the higher temperature is raised. Such interpretation of anisotropic line broadening has been recently applied in our group for studying ball-milled nanocrystalline fluorides $\mathrm{MF}_{2}(\mathrm{M}=\mathrm{Sr}, \mathrm{Ca}, \mathrm{Ba}, \mathrm{Cd})$ with the fluorite-type crystal structure from which the deformation behaviour of the materials were carefully pointed out from diffraction line broadening analysis [42].
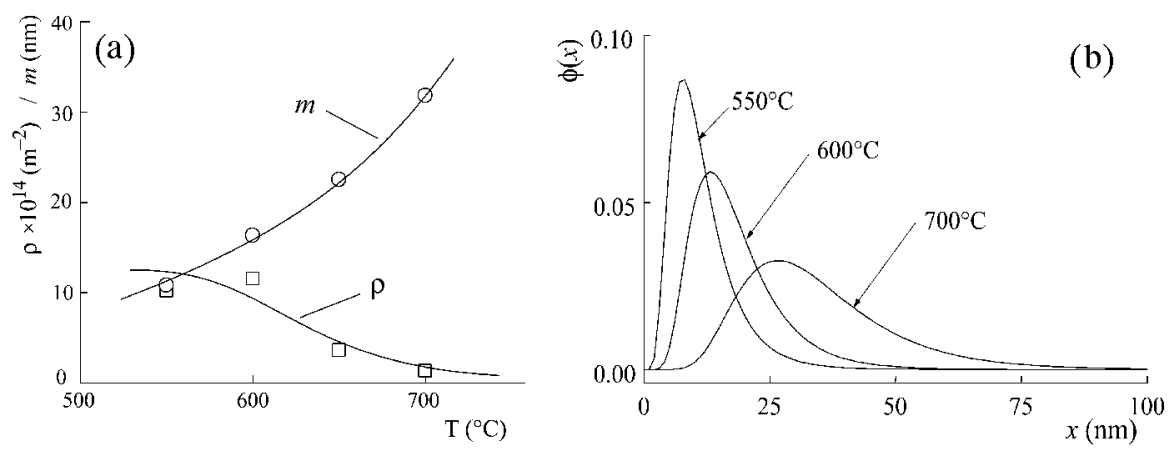

Fig. 3. Microstructure characteristics of nanocrystalline $\mathrm{CeO}_{2}$ prepared at different temperatures (from Ref. [34]). (a) Dislocation density $\rho$ and mean values $m$ of the size distribution functions; (b) size distribution functions of $\mathrm{CeO}_{2}$. 


\section{Conclusions}

To conclude, nanocrystalline materials can be characterized from diffraction line profile analysis in terms of crystallite size, distribution, dislocations, and stacking faults. The presence of more than one effect can make the study more complicated and limited. However, examples have been reported, in which the microstructural parameters were nicely correlated to chemical or physical properties, such as for example the enhancement of electrochemical properties from mechanically-activated nanocrystalline nickel hydroxide nitrate [43]. Diffraction methods for the study of nanocrystalline materials are still in progress and new developments can be expected in the near future of the use of whole pattern modelling based on the physical parameter approach.

\section{References}

[1] H. Gleiter, J. Appl. Crystallogr. 24, 79 (1991).

[2] L. Spanhel, M.A. Anderson, J. Am. Chem. Soc. 113, 2826 (1991).

[3] Y. Wang, N. Herron, Phys. Rev. B 42, 7253 (1990).

[4] J.I. Langford, D. Louër, Rep. Prog. Phys. 59, 131 (1996).

[5] D. Louër, Acta Crystallogr. A 54, 922 (1998).

[6] D. Louër, J.P. Auffrédic, J.I. Langford, D. Ciosmak, J.C. Niepce, J. Appl. Crystallogr. 16, 183 (1983).

[7] T. Ungár, A. Borbély, Appl. Phys. Lett. 69, 3173 (1996).

[8] P. Scardi, Y.H. Dong, M. Leoni, Mater. Sci. Forum, in press.

[9] J. Pielaszek, X-Ray Diffractometry in Supported Catalysts Studies, Polska Akad. Nauk, Instytut Chemii Fizycznej, Warszawa 1995.

[10] R.L. Snyder, J. Fiala, H.J. Bunge, Defect and Microstructure Analysis by Diffraction, IUCr/OUP, Oxford 1999.

[11] D. Louër, N. Audebrand, Adv. X-ray Anal. 41, 556 (1999).

[12] Y.H. Dong, P. Scardi, J. Appl. Crystallogr. 33, 184 (2000).

[13] P. Thompson, D.E. Cox, J.B. Hastings, J. Appl. Crystallogr. 20, 79 (1987).

[14] P.W. Stephens, J. Appl. Crystallogr. 32, 281 (1999).

[15] R. Delhez, T.H. de Keijser, J.I. Langford, D. Louër, E.J. Mittemeijer, E.J. Sonneveld, in: The Rietveld Method, Ed. R.A. Young, IUCr/OUP, Oxford 1995 , p. 132.

[16] P. Scardi, M. Leoni, J. Appl. Crystallogr. 32, 671 (1999).

[17] A. Guinier, Théorie et technique de la radiocristallographie, Dunod, Paris 1956.

[18] A.J.C. Wilson, X-Ray Optics, Methuen, London 1962.

[19] E.F. Bertaut, Acta Crystallogr. 3, 14 (1950).

[20] J.I. Langford, in: Accuracy in Powder Diffraction, Spec. Publ. 846, Eds. E. Prince, J.K. Stalick, NIST, Gaithersburg 1992, p. 110.

[21] J.I. Langford, D. Louër, J. Appl. Crystallogr. 15, 20 (1982). 
[22] B.E. Warren, X-Ray Diffraction, Addison-Wesley, Reading 1969.

[23] A.R. Stokes, A.J.C. Wilson, Proc. Phys. Soc. Lond. 56, 174 (1944).

[24] J.I. Langford, R. Delhez, T.H. de Keijser, E.J. Mittemeijer, Aust. J. Phys. 41, 173 (1988).

[25] T. Ungár, G. Tichy, Phys. Status Solidi A 171, 425 (1999).

[26] P. Klimanek, R. Kužel, J. Appl. Crystallogr. 21, 59 (1988).

[27] M.A. Krivoglaz, Theory of X-Ray and Thermal Neutron Scattering by Real Crystals, Plenum Press, New York 1969.

[28] M. Wilkens, Phys. Status Solidi A 2, 359 (1970).

[29] T. Ungár, I. Dragomir, Á. Révész, A. Borbély, J. Appl. Crystallogr. 32, 992 (1999).

[30] G. Ribárik, T. Ungár, J. Gubicza, J. Appl. Crystallogr., in press.

[31] J.I. Langford, D. Louër, P. Scardi, J. Appl. Crystallogr. 33, 964 (2000).

[32] J.I. Langford, A. Boultif, J.-P. Auffrédic, D. Louër, J. Appl. Crystallogr. 26, 22 (1993).

[33] G.K. Williamson, W.H. Hall, Acta Metall. 1, 22 (1953).

[34] T. Ungár, I. Dragomir-Cernatescu, D. Louër, N. Audebrand, J. Phys. Chem. Solids 62, 1935 (2001).

[35] N. Guillou, J.P. Auffrédic, D. Louër, Powder Diffr. 10, 236 (1995).

[36] N. Audebrand, J.-P. Auffrédic, D. Louër, Chem. Mater. 12, 1791 (2000).

[37] N. Guillou, L.C. Nistor, H. Fuess, E. Hahn, Nanostruct. Mater. 8, 545 (1997).

[38] J.-P. Auffrédic, A. Boultif, J.I. Langford, D. Louër, J. Am. Ceram. Soc. 78, 323 (1995).

[39] D. Louër, R. Vargas, J.P. Auffrédic, J. Am. Ceram. Soc. 67, 136 (1984).

[40] N. Audebrand, J.P. Auffrédic, D. Louër, Chem. Mater. 10, 2450 (1998).

[41] N. Audebrand, D. Louër, J. Phys. IV (France) 8, 109 (1988).

[42] H. Palancher, D. Louër, N. Audebrand, G. Ribárik, T. Ungár, J. Appl. Crystallogr., submitted for publication.

[43] D. Louër, A. De Guibert, J. Mater. Sci. 20, 3729 (1985). 